\title{
Mental health of healthcare workers during the COVID-19 pandemic in Italy
}

\author{
Marialaura Di Tella ${ }^{1}$, Annunziata Romeo ${ }^{2}$, Agata Benfante $^{1}$, and Lorys Castelli ${ }^{1}$ \\ ${ }^{1}$ Affiliation not available \\ ${ }^{2}$ University of Turin
}

May 6, 2020

\begin{abstract}
Background: The COVID-19 pandemic represents one of the most stressful events of recent times. Among the population, healthcare professionals who treat COVID-19 patients are most likely to develop psychological distress and posttraumatic stress symptoms (PTSS). The present study thus aimed to investigate the psychological impact of the COVID-19 outbreak on Italian healthcare workers. Methods: The responses of 145 healthcare workers (72 medical doctors and 73 nurses) were included in the final dataset. Participants were asked to provide sociodemographic and clinical information, and to complete: 1) quality of life and health-related Visual Analogue Scales, 2) State-Trait Anxiety Inventory-Form Y1, 3) Beck Depression Inventory, and 4) PTSD Checklist for DSM-5. Results: A comparison between healthcare professionals working in COVID-19 wards and other units revealed that the former reported higher levels of both depressive symptoms and PTSS. Moreover, the results of regression analyses showed that in healthcare professionals working with COVID-19 patients, gender and marital status, and gender and age significantly predicted depressive symptoms and PTSS, respectively. Particularly, being female and not in a relationship were found to be associated with higher levels of depressive symptoms, whereas being female and older were found to be related to higher levels of PTSS. Conclusions: The current findings suggest that specific predisposing factors could identify healthcare workers who are at high risk of developing mental health symptoms when faced with COVID-19 patients.
\end{abstract}

\section{INTRODUCTION}

The COVID-19 pandemic represents one of the most stressful events in recent times worldwide. This pandemic poses a major challenge to social, economic, and, above all, the psychological resources of the population. Undoubtedly, healthcare workers are the most exposed category. Their psychological burden is likely due to the staff reorganisation, the working intensity, and the anxiety of being exposed to the virus at hospital and, in turn, of bringing the infection home. ${ }^{1}$ The healthcare workers directly involved in the care of patients with COVID-19 are at high risk of developing psychological distress and other mental health symptoms such as posttraumatic stress symptoms (PTSS). ${ }^{2}$

The main aim of the present study was to investigate the psychological impact of the COVID-19 outbreak on Italian healthcare workers. First, we compared the levels of psychological distress (i.e., anxiety/depressive symptoms) and PTSS between healthcare professionals working in the front line of COVID-19 and healthcare professionals working in other units. Second, we explored which sociodemographic and clinical factors could significantly predict psychological distress and PTSS levels in the group of healthcare workers involved in the care of COVID-19 patients.

\section{METHODS}

The data were collected using an online survey from March 19 to April 5, 2020. An anonymised, individual, and unique code to complete the survey was provided to those who agreed to participate in the study. 
The responses of 145 healthcare workers (72 medical doctors and 73 nurses) were included in the final dataset. Participants were asked to provide sociodemographic and clinical information, and to complete: 1) quality of life and health-related Visual Analogue Scales (VAS); 2) State-Trait Anxiety Inventory-Form Y1 (STAI Y1); 3) Beck Depression Inventory (BDI-II); and 4) PTSD Checklist for DSM-5 (PCL-5) to investigate the presence of PTSS.

The study was approved by the University of Turin Ethics Committee and conducted in

accordance with the Declaration of Helsinki. All the participants gave their written informed

consent to participate in the study.

\section{RESULTS}

The total sample had a mean age of $42.90(\mathrm{SD}=11.20)$ years, $72 \%(105)$ of participants were female, and $43 \%$ (63) worked in COVID-19 wards. The majority of the participants had at least one child $(56 \%, 81)$ and were in a relationship $(61 \%, 88)$.

The group comparisons of sociodemographic and clinical characteristics, quality of life and health-related data, and psychological measures (BDI, STAI Y2, and PCL-5) are presented in Fig. 1 .

The results of comparisons showed that healthcare professionals who work in COVID-19 wards were younger $($ mean \pm SD: $39.05 \pm 8.28$ vs. $45.87 \pm 12.24, t(140.91)=-3.993, p<.001, d=0.65)$ and more likely not in a relationship $\left(\chi^{2}(1)=4.573, p=.032\right)$, compared to healthcare professionals working in other facilities.

With regard to the psychological assessment, healthcare professionals who work COVID-19 wards reported higher levels of both depressive symptoms $(p=.005, d=0.40)$ and PTSS $(p=.015, d=0.47)$ compared to healthcare professionals working in non-COVID-19 facilities. A chi-square test confirmed the presence of a significant association between participants who scored above the PCL-5 cut-off ([?] 33) and those who worked in COVID-19 wards $\left(\chi^{2}(1)=4.374, p=.036\right)$.

Considering the high levels of depressive symptoms and PTSS reported by healthcare professionals who work COVID-19 wards, two multiple regression analyses were performed to assess whether sociodemographic and clinical variables (age, gender, having/not having children, being/not being in a relationship, having/not having a medical condition) significantly predicted the BDI and PCL-5 scores in this subgroup of healthcare workers.

With regard to depressive symptoms, the multiple regression model statistically significantly predicted the BDI total score, $F(5,57)=4.970, p=.001$, adj. $R^{2}=.24$. Among the five predictors, gender $(\beta=-0.252$, $p=.032)$ and marital status $(\beta=0.441, p=.001)$ were statistically significant. Particularly, being female and not in a relationship were found to be associated with higher levels of depressive symptoms.

Regarding PTSS, the multiple regression model statistically significantly predicted the PCL-5 total score, $F$ $(5,57)=5.127, p=.001$, adj. $R^{2}=.25$. Among the five predictors, age $(\beta=0.554, p=.001)$ and gender $(\beta=-0.398, p=.001)$ were statistically significant. Particularly, being female and older were found to be related to higher levels of PTSS.

\section{DISCUSSION}

The present results showed that healthcare professionals who work in COVID-19 wards reported higher levels of depressive symptoms and PTSS than those who work in other healthcare units. These findings are very understandable in view of the fact that healthcare workers involved in the frontline of COVID-19 are in a daily struggle to keep the patients alive. Furthermore, medical health workers constantly find themselves exposed to highly risky situations, which can make them feel unsafe at work. The relentless spread of the virus, the lack of sufficient rest, the permanent threat of being infected, the workload, the shortage of medical protective equipment, and frequent isolation from family are all factors that can contribute to the high risk of acute mental health conditions in healthcare workers in the long term. ${ }^{4-6} \mathrm{In}$ particular, the prolonged 
exposure to traumatic events, such as the death of patients and colleagues, may lead to the development of severe anxiety and depressive symptoms, as well as PTSS. ${ }^{2,3}$

Possible predisposing factors for the mental health symptoms observed in healthcare professionals working in COVID-19 wards were found to be being female and not in a relationship for depressive symptoms and being female and older for PTSS.

With regard to gender differences, our findings are in line with previous studies ${ }^{2,3}$ that showed a higher prevalence of PTSS in women than men, in both general population and healthcare workers. Regarding age differences, the available evidence is still controversial and, in some cases, no differences ${ }^{3}$ or opposite results have been obtained ${ }^{2}$. For instance, in contrast to our data, the study of Tang et al. ${ }^{2}$ showed that the healthcare workers aged between 20 and 30 years obtained the highest PTSS scores during a previous pandemic. Conversely, Liu et al. ${ }^{3}$ found that in the general population, the youngest reported the lowest level of PTSS, but age did not represent a predictive factor for developing PTSS during the COVID-19 epidemic. Furthermore, it is well known that women are more vulnerable to developing depressive symptoms ${ }^{7,8}$ and that adequate social support provided by partners or spouses represents a protective factor for the psychological well-being. ${ }^{9,} 10$

Taken together, our findings suggest that healthcare professionals involved in COVID-19 management display high levels of both depressive symptoms and PTSS. Moreover, specific predisposing factors seem to characterise those healthcare worker members who are at high risk of developing depressive symptoms and PTSS when faced with COVID-19 patients.

During the extraordinary events associated with the COVID-19 pandemic, it is essential to take on healthcare workers' needs, providing timely psychosocial and mental health support, particularly for those groups identified at risk. Indeed, healthcare professionals expect to be welcomed, listened to, supported, and protected by their organisations. ${ }^{1}$

\section{References}

1. Shanafelt T, Jonathan R, Mickey T. Understanding and addressing sources of anxiety among health care professionals during the COVID-19 pandemic. JAMA 2020. doi:https://doi.org/10.1001/jama.2020.5893.

2. Tang L, Pan L, Yuan L, Zha L. Prevalence and related factors of post-traumatic stress disorder among medical staff members exposed to H7N9 patients. Int J Nurs Sci 2017;4(1):63-67. doi:http://dx.doi.org/10.1016/j.ijnss.2016.12.002

3. Liu N, Zhang F, Wei C, et al. Prevalence and predictors of PTSS during COVID-19 outbreak in China hardest-hit areas: Gender differences matter. Psychiatry Res 2020;187:112921. doi:10.1016/j.psychres.2020.112921

4. Zhang WR., Wang K, Yin L, et al. Mental health and psychosocial problems of medical health workers during the COVID-19 epidemic in China. Psychother Psychosom 2020;1-9. doi:https://doi.org/10.1159/000507639

5. Neto MLR, Almeida HG, Esmeraldo JDA, et al. When health professionals look death in the eye: the mental health of professionals who deal daily with the 2019 coronavirus outbreak. Psychiatry Res 2020;112972. doi:10.1016/j.psychres.2020.112972

6. Lai J, Ma S, Wang Y, et al. Factors associated with mental health outcomes among health care workers exposed to coronavirus disease 2019. JAMA Netw Open 2020;3(3):e203976-e203976. doi:10.1001/jamanetworkopen.2020.3976

7. Piccinelli M, Wilkinson G. Gender differences in depression: Critical review. Br J Psychiatry 2000;177(6):486-492. doi:10.1192/bjp.177.6.486

8. Irish LA, Fischer B, Fallon W, Spoonster E, Sledjeski EM, Delahanty DL. Gender differences in PTSD symptoms: an exploration of peritraumatic mechanisms. J Anxiety Disord 2011;25(2):209-216. doi:10.1016/j.janxdis.2010.09.004

9. Braithwaite S, Holt-Lunstad J. Romantic relationships and mental health. Curr Opin Psychol 
2017;13:120-125. doi:https://doi.org/10.1016/j.copsyc.2016.04.001

10. Dush CMK, Amato PR. Consequences of relationship status and quality for subjective well-being. J Soc Pers Relatsh 2005;22(5):607-627. doi:https://doi.org/10.1177/0265407505056438

\section{Conflict of Interest}

The authors declare that they have no conflicts of interest.

\section{Ethical Approval}

The study was approved by the University of Turin Ethics Committee (Prot. n. 142069) and was conducted in accordance with the Declaration of Helsinki. All the participants gave their written informed consent to participate in the study.

Fig. 1. Group comparisons on sociodemographic and clinical data, quality of life and healthrelated items, and psychological outcomes of healthcare professionals working in COVID-19 wards vs other wards $(\mathbf{N}=\mathbf{1 4 5})$. (A) Percentages of healthcare workers based on gender, marital status, children, and medical condition. (B) Mean (SE) scores of healthcare workers for the three Visual Analog Scales (0-10), assessing health concern, health evaluation, and life satisfaction.(C) Mean (SE) scores of healthcare workers for the State-Trait Anxiety Inventory (STAI-Y1, range 20-80), Beck Depression Inventory (BDI-II, range 0-55), and Posttraumatic Stress Disorder Checklist for DSM-5 (PCL-5, range 0-80).

Note: ${ }^{*}$ p-value $<.05 ; * *$ p-value $<.01$.

\section{Hosted file}

Figure.docx available at https://authorea.com/users/318889/articles/448728-mental-health-ofhealthcare-workers-during-the-covid-19-pandemic-in-italy 